\title{
Involuntary sustained firing of plantar flexor motor neurones: effect of electrical stimulation parameters during tendon vibration
}

\author{
Ricardo N. O. Mesquita ${ }^{1}$ (D) . Janet L. Taylor ${ }^{1,2}$ (D) Benjamin Kirk ${ }^{1}$ (D) - Anthony J. Blazevich ${ }^{1}$ (D)
}

Received: 20 July 2020 / Accepted: 16 November 2020 / Published online: 3 January 2021

(c) The Author(s) 2021

\begin{abstract}
Purpose Simultaneous application of tendon vibration and neuromuscular electrical stimulation (NMES) induces an involuntary sustained torque. We examined the effect of different NMES parameters (intensity, pattern of stimulation and pulse width) on the magnitude of the evoked involuntary torque.

Methods Plantar flexor torque was recorded during 33-s Achilles tendon vibration with simultaneous 20-Hz NMES bouts on triceps surae ( $n=20 ; 13$ women). Intensity was set to elicit 10,20 or 30\% of maximal voluntary contraction torque (MVC), pulse width was narrow $(0.2 \mathrm{~ms})$ or wide $(1 \mathrm{~ms})$, and the stimulus pattern varied $(5 \times 2-\mathrm{s}$ or $10 \times 1-\mathrm{s})$. Up to 12 different trials were performed in a randomized order, and then repeated in those who produced a sustained involuntary torque after the cessation of vibration.

Results Six of 7 men and 5 of 13 women produced a post-vibration sustained torque. Eight of 20 participants did not complete the $30 \%$ trials, as they were perceived as painful. Torque during vibration at the end of NMES and the increase in torque throughout the trial were significantly higher in 20 than $10 \%$ trials $(n=11 ; 9.7 \pm 9.0$ vs $7.1 \pm 6.1 \%$ MVC and $4.3 \pm 4.5$ vs $3.6 \pm 3.5 \% \mathrm{MVC}$, respectively). Post-vibration sustained torque was higher in wide pulse-width trials $(5.4 \pm 5.9$ vs $4.1 \pm 4.3 \%$ MVC). Measures of involuntary torque were not different between 20 and $30 \%$ trials $(n=8)$.

Conclusion Bouts of $5 \times 2$-s NMES with wide pulse width eliciting $20 \%$ MVC provides the most robust responses and could be used to maximise the production of involuntary torque in triceps surae.
\end{abstract}

Keywords Motor neurone $\cdot$ Central torque $\cdot$ Bistability $\cdot$ Serotonin $\cdot$ Noradrenaline

$\begin{array}{ll}\text { Abbreviations } \\ \text { CV } & \text { Coefficient of variation } \\ \text { EMG } & \text { Electromyographic } \\ \text { ICC } & \text { Intraclass correlation coefficient } \\ \text { MU } & \text { Motor unit } \\ \text { NMES } & \text { Neuromuscular electrical stimulation } \\ \text { MDC } & \text { Minimum detectable change } \\ \text { MNs } & \text { Spinal motor neurones } \\ \text { MVC } & \text { Maximal isometric voluntary plantar flexor } \\ & \text { contraction } \\ \text { PICs } & \text { Persistent inward currents }\end{array}$

Communicated by Toshio Moritani.

Ricardo N. O. Mesquita

r.mesquita@ecu.edu.au

1 Centre for Exercise and Sports Science Research, School of Medical and Health Sciences, Edith Cowan University, Perth, Australia

2 Neuroscience Research Australia, Sydney, Australia

$\begin{array}{ll}\text { TE } & \text { Typical error } \\ T_{\text {sust }} & \text { Self-sustained torque } \\ T_{\text {vib }} & \text { Reflexive torque during vibration } \\ \text { WU } & \text { Warm-up } \\ \eta_{\mathrm{p}}{ }^{2} & \text { Partial eta-squared }\end{array}$

\section{Introduction}

Neuromuscular electrical stimulation (NMES) and tendon vibration have been used independently (e.g. Magalhães et al. 2013; Bochkezanian et al. 2018b) and simultaneously (Magalhães and Kohn 2010; Trajano et al. 2014; Bochkezanian et al. 2018a; Kirk et al. 2019) to induce motor unit (MU) activation, and hence muscle contractions. This involuntary MU activation is induced via both peripheral and central pathways. NMES depolarizes both motor and sensory axons (Bergquist et al. 2011b), inducing direct MU recruitment with signals travelling from the stimulation site to the muscle as well as an indirect MU recruitment with 
signals travelling through the spinal motor neurones (MNs). Tendon vibration imposes repetitive, small muscle-length changes that stimulate muscle spindles, inducing excitatory drive primarily from Ia-afferents onto the $\mathrm{MN}$ and provoking a reflexive activation of MUs (Grande and Cafarelli 2003).

In previous studies, an NMES-induced involuntary "extra torque" that is larger than would be expected from the direct activation of motor axons has been observed (e.g. Collins et al. 2001; Wegrzyk et al. 2015). The contribution of peripheral (i.e. within the muscle) mechanisms to this phenomenon is controversial, given that this "extra torque" was abolished during anaesthesia of the motor axons proximal to the stimulation site in some (Collins et al. 2001; Blouin et al. 2009; Lagerquist et al. 2009) but not all (Frigon et al. 2011) studies. Other empirical evidence corroborates the contribution of centrally-mediated mechanisms. This evidence includes greater levels of "extra torque" (Collins et al. 2002; Lagerquist and Collins 2010) and electromyographic (EMG) activity (Arpin et al. 2019) during wide- than narrow-pulse NMES, and the enhancement of H-reflexes, V- and F-wave amplitudes in association with extra torque development during NMES (Klakowicz et al. 2006; Lagerquist and Collins 2010; Bergquist et al. 2011a; Vitry et al. 2019) but also during tendon vibration (Magalhães et al. 2013) or both simultaneously (Magalhães and Kohn 2010). The relative contribution of peripheral and central pathways to this phenomenon has mainly been discussed in relation to NMES, and a greater central contribution is expected when combining both NMES and tendon vibration. Tendon vibration per se recruits low-threshold MUs (Grande and Cafarelli 2003) and the superposition of bursts of NMES may activate additional afferent fibres and consequently activate a greater proportion of the MU pool (Collins et al. 2001).

The central mechanisms commonly suggested to explain the occurrence of "extra torque" are a presynaptic development of post-tetanic potentiation of neurotransmitter release due to a repetitive depolarization of Ia afferents (Hirst et al. 1981) and/or postsynaptic activation of persistent inward currents (PICs). PICs are a strong intrinsic MN property activated via voltage-dependent channels on the MN dendrites (Hounsgaard and Kiehn 1993; Lee and Heckman 1996) and modulated by serotonergic and noradrenergic drive onto the MN (Lee and Heckman 2000). They amplify and prolong the effects of synaptic input (Heckman et al. 2005) by providing a sustained depolarising current to the MNs, accelerating initial MN firing and contributing to the repetitive firing required for muscle contractions (Heckman et al. 2008). As PICs are initiated by excitatory synaptic signals onto the MN, they can be initiated by excitatory afferent input (Heckman et al. 2008). Some features of responses to combined tendon vibration and NMES are consistent with the behavior of PICs. Self-sustained torque observed after the end of such trials (or after vibration alone; Gorassini et al. 2002) could be explained by PIC-related bistable behavior in some spinal MNs (Lee and Heckman 1998). The progressive increase of torque reported during vibration plus NMES (Magalhães and Kohn 2010; Trajano et al. 2014; Kirk et al. 2019) is similar to the warm-up effect that is thought to reflect depolarization-induced facilitation of voltage-gated calcium channels (Svirskis and Hounsgaard 1997). Finally, the "extra torque" is joint angle (i.e. muscle length) dependent (Trajano et al. 2014), consistent with the effect of reciprocal inhibition shown in vivo using voltage clamp techniques (Hyngstrom et al. 2007). Thus, it is possible that responses to combined tendon vibration and NMES are explicable by PIC activity, although a robust examination of these behaviors remains to be conducted to determine whether PIC strength can be estimated in vivo using this technique.

The magnitude of involuntary torque produced during simultaneous tendon vibration and NMES may hypothetically allow the indirect estimation of PIC behavior, presumably in a relatively large number of motor units in the absence of voluntary corticospinal drive and using equipment that is available in many laboratories. It might therefore prove to be a useful alternative to the paired motor unit technique (Gorassini et al. 2002), the standard method to indirectly estimate PIC strength in humans. Nonetheless, the technique under examination in this study has been rarely used in research and the specific test parameters that might provide the greatest involuntary torque have not been determined. These parameters should be optimized before further investigations are conducted. Whilst the tendon vibration parameters that might produce significant Ia traffic are largely known, it is currently unclear how NMES parameters such as stimulus intensity, pattern of stimulation and pulse width affect the test results. Therefore, the aim of the present study was to systematically test the effects of different NMES parameters (intensity, pattern of stimulation and pulse width) on the magnitude of the evoked involuntary torque.

\section{Methods}

\section{Participants}

Twenty healthy adults ( 7 men and 13 women; age: $28.0 \pm 5.1$ years; body mass: $68.6 \pm 15.9 \mathrm{~kg}$; height: $169.8 \pm 11.1 \mathrm{~cm}$ ) volunteered for the study. Exclusion criteria included diagnosed neurological disorders, current acute or chronic injuries, and medications that might affect central monoamine concentrations. Participants were fully informed of any risks or discomforts associated with the procedures before giving their written informed consent to participate. The procedures were approved by the University Human 
Research Ethics Committee of Edith Cowan University, and performed according to the Declaration of Helsinki, except for registration in a database.

\section{Procedures}

Data collection was performed in a single session and participants were asked to abstain from caffeine-containing foods and beverages within $12 \mathrm{~h}$ of the experimental session. Participants were seated in the chair of an isokinetic dynamometer (Biodex System 4, Biodex Medical System, USA) with the hips at $50^{\circ}$ of flexion $\left(0^{\circ}=\right.$ extended neutral position), right knee fully extended $\left(0^{\circ}\right)$ and right ankle at $10^{\circ}$ of dorsiflexion $\left(0^{\circ}=\right.$ neutral position $)$. The right foot was tightly fixed to the plantar flexor attachment of the dynamometer and the axis of rotation was aligned with the lateral malleolus. The distance between the chair and the foot attachment was adjusted to find the minimum distance at which participants were able to straighten their right leg, to minimise the elevation of the heel during plantar flexions. Participants were then instructed to perform four isometric plantar flexion contractions at 20,40,60 and $80 \%$ of perceived maximal effort as warm-up and task familiarization. The participants subsequently performed at least three maximal isometric voluntary plantar flexor contractions (MVC) with a 90-s inter-trial passive rest; additional trials were performed if they reached a torque value $>5 \%$ higher than the previous best attempt. Visual plantar flexor torque feedback was continuously provided on a large screen in front of the participant, and standardized verbal encouragement was provided by the same investigator in all trials. The peak isometric plantar flexor torque was taken as the highest value during the MVCs and used to set NMES current intensities. After 3 min of rest, $0.5-\mathrm{s} 20-\mathrm{Hz}$ trains of NMES were applied percutaneously to the plantar flexors at increasing intensities to determine the current required to evoke 10 , 20 and $30 \%$ of MVC torque with both narrow $(0.2 \mathrm{~ms})$ and wide (1 ms) pulse widths. Subsequently, NMES was applied in 1- and 2-s bursts with both narrow and wide pulse widths at the pre-determined current intensities during a period of ongoing Achilles tendon vibration in different trials. An initial set of up to 12 trials was conducted, and in each trial a different combination of NMES intensities, pulse widths and patterns was imposed in a randomized order. A warmup effect during the trial (i.e. increased torque produced in successive stimulations) and self-sustained (ongoing, involuntary) torque following the trial was observable in 11 out of 20 participants. These "responders" then performed a 2nd equivalent block after a 10-min rest. Eight of the 20 participants perceived the intensity to elicit $30 \% \mathrm{MVC}$ torque as painful (including 3 of 11 "responders") and therefore trials at this intensity were not conducted for these participants, with only eight trials being performed. Two minutes of rest was provided between trials (Fig. 1).

\section{Tendon vibration with superimposed NMES}

This technique combines tendon vibration and percutaneous NMES to provoke involuntary muscle contractions (Kirk et al. 2019; Magalhães and Kohn 2010; Trajano et al. 2014). The present study was designed to systematically manipulate the NMES parameters used with this technique to determine their influence on involuntary torque variables. In each trial, the Achilles tendon was mechanically vibrated at $115 \mathrm{~Hz}$ (1-mm deflections) for $33 \mathrm{~s}$ by a hand-held vibrator (Vibrasens, Techno Concepts,

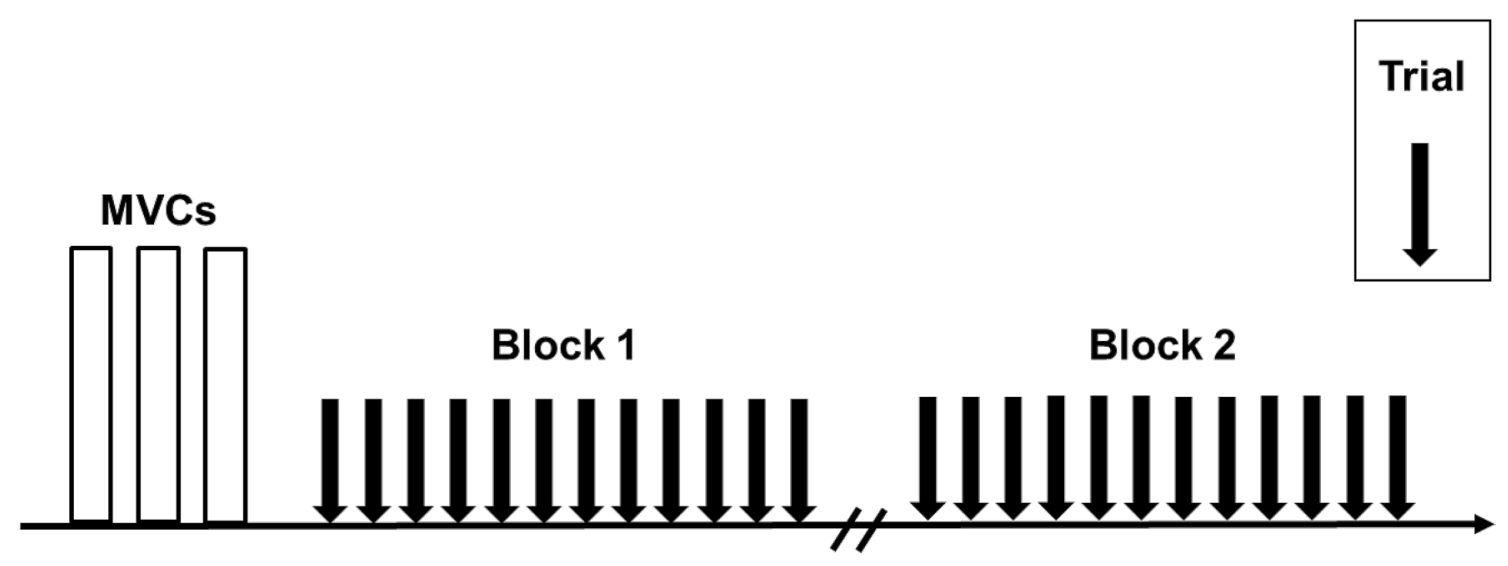

Fig. 1 Experimental protocol. After performing maximal voluntary contractions (MVCs) of plantar flexors, a set of up to 12 trials (Block 1) was completed during which plantar flexor neuromuscular electrical stimulation (NMES) was superimposed on Achilles tendon mechanical vibration. A 2 nd equivalent block was performed after a 10-min passive rest in those participants who produced a sustained involuntary torque after the cessation of vibration in the trials of Block 1. Between trials, current intensity (to evoke 10, 20 and $30 \%$ of MVC torque), pulse width ( $0.2 \mathrm{~ms}$ vs $1 \mathrm{~ms})$ and pattern of stimulation $(5 \times 2$-s bursts at 4 -s intervals vs $10 \times 1$-s bursts at 2 -s intervals) were varied randomly 
France). The vibrator was held with a steady pressure by the same researcher on the posterior aspect of the Achilles tendon at the level of the medial malleolus. After $10 \mathrm{~s}$ of vibration, trains of NMES at a $20-\mathrm{Hz}$ frequency were applied to the triceps surae whilst tendon vibration continued. A constant-current electrical stimulator (DS7, Digitimer Ltd, United Kingdom) was used to deliver electrical square-wave stimuli through two self-adhesive electrodes $(5 \times 9 \mathrm{~cm}$; Dura-Stick Plus, DJO Global, USA), with the cathode placed transversely and distal to the popliteal crease and the anode transversely and over the distal gastrocnemius-Achilles muscle-tendon junction. NMES parameters were varied between trials, with a total of 12 possible combinations. Two pulse widths $(0.2$ and $1 \mathrm{~ms})$, three current intensities (to evoke 10, 20 and 30\% of MVC torque) and two patterns of stimulation $(5 \times 2$-s bursts at 4 -s intervals and $10 \times 1$-s burst at 2 -s intervals) were imposed. During the trials, the participants were instructed to hold the shoulder straps of the chair, to look forward at the blank (black) display monitor, silently count backwards from 50 by one, to remain quiet, and to not voluntarily activate their leg muscles.

Three torque-related quantities were calculated (Fig. 2) and presented as a percentage of MVC.

Reflexive torque during vibration $\left(T_{\mathrm{vib}}\right)$ : mean torque in a 500-ms window starting $500 \mathrm{~ms}$ after the cessation of NMES, but during tendon vibration.

Self-sustained torque $\left(T_{\text {sust }}\right)$ : mean torque in a $500-\mathrm{ms}$ window starting $3 \mathrm{~s}$ after the cessation of vibration.

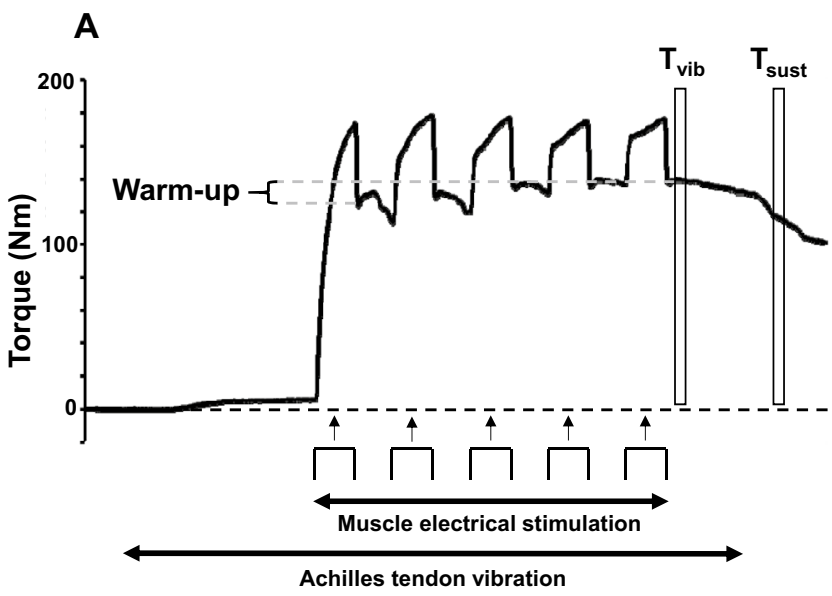

Fig. 2 Data from two different trials from the same participant to illustrate the torque response to tendon vibration with superimposed neuromuscular electrical stimulation (NMES). In each trial, the Achilles tendon was mechanically vibrated at $115 \mathrm{~Hz}$ for $33 \mathrm{~s}$. After $10 \mathrm{~s}$ of vibration, NMES $(20 \mathrm{~Hz})$ was applied to the triceps surae whilst tendon vibration continued. Torque produced during a test using $5 \times 2$-s bursts at 4 -s intervals ( 2 -s on, 2 -s off) with a wide (1-ms) pulse width and at an intensity that evoked a torque of $20 \%$
Warm-up: the difference between $T_{\mathrm{vib}}$ and the mean torque in a $500-\mathrm{ms}$ window starting $500 \mathrm{~ms}$ after the first burst of NMES.

\section{Statistical analysis}

Data are presented as mean \pm standard deviation (mean \pm SD). Statistical analyses were performed using IBM SPSS Statistics (v25, SPSS Inc., USA). Normality of the data was examined using Shapiro-Wilk test. Warm-up effect, $T_{\text {vib }}$ and $T_{\text {sust }}$ were subjected to separate 2 (Intensity: $10 \mathrm{vs}$ $20 \%$ MVC) $\times 2$ (Pulse width: wide vs narrow) $\times 2$ (Pattern: $5 \times 2$-s vs $10 \times 1$-s bursts) $\times 2$ (Block: block 1 vs block 2$)$ repeated measures ANOVA. Given that not all participants performed 30\% MVC trials, statistical analysis was initially conducted without the $30 \% \mathrm{MVC}$ condition $(n=11)$. Additional ANOVAs with the inclusion of this intensity were also conducted $(n=8)$. For all ANOVAs, sphericity was assessed using Mauchly's test of sphericity; Greenhouse-Geisser correction was used when sphericity was violated. The Least Significant Difference method was used for post-hoc comparisons when up to three means were compared, whilst a false discovery rate analysis was used when more than three means were compared to minimise the risk of type I error resulting from multiple pairwise comparisons. Effect sizes are reported as partial eta squared $\left(\mathrm{n}_{\mathrm{p}}{ }^{2}\right)$. Statistical significance was set at an alpha level of 0.05 .

Follow-up pairwise analysis of trials for reliability was performed for the combination of electrical parameters which yielded the greatest magnitude of response. The

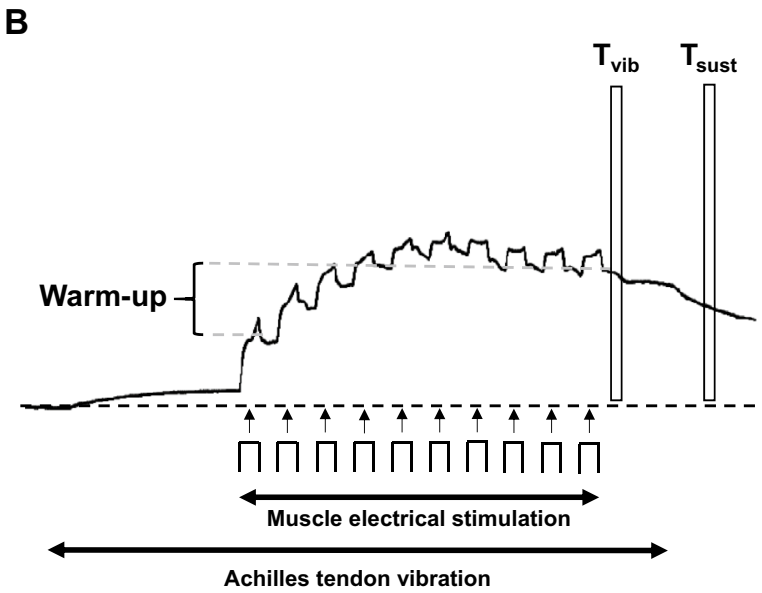

of maximal voluntary contraction (MVC) torque is shown in A, and torque produced using $10 \times 1$-s bursts at 2 -s intervals (1-s on, 1-s off) with a narrow (0.2-ms) pulse-width and at an intensity that evoked a torque of $10 \%$ MVC is shown in B. Current intensity, pulse width and pattern of stimulation were altered in each trial. $T_{\mathrm{vib}}$ torque measured during vibration after the last burst of NMES, $T_{\text {sust }}$ torque measured $3 \mathrm{~s}$ after vibration cessation (self-sustained torque), Warm-up difference between $\mathrm{T}_{\mathrm{vib}}$ and torque after the first burst of NMES 
level of reliability of the involuntary torque variables was determined when assessed in different sets (i.e. Set 1 vs Set 2). The indices of reliability that were calculated were twoway mixed effects intraclass correlation coefficients (ICC 3,1 ), typical error (TE; i.e. standard error of measurement) through a freely available spreadsheet (Hopkins 2017), minimum detectable change (MDC) through the formula $\mathrm{MDC}=\mathrm{TE} \times 1.96 \times \sqrt{ } 2$, and intra-individual coefficient of variation $(\mathrm{CV})$ as the average of the $\mathrm{CV}$ s for each individual.

\section{Results}

Both a warm-up effect during the trial and self-sustained torque following vibration cessation were observed in 11 out of 20 participants (6 of 7 men and 5 of 13 women). These 11 participants were designated as "responders" and formed the study cohort. Trials with NMES intensity of $30 \%$ MVC were not performed by all participants, as 8 out

\section{Block 1}

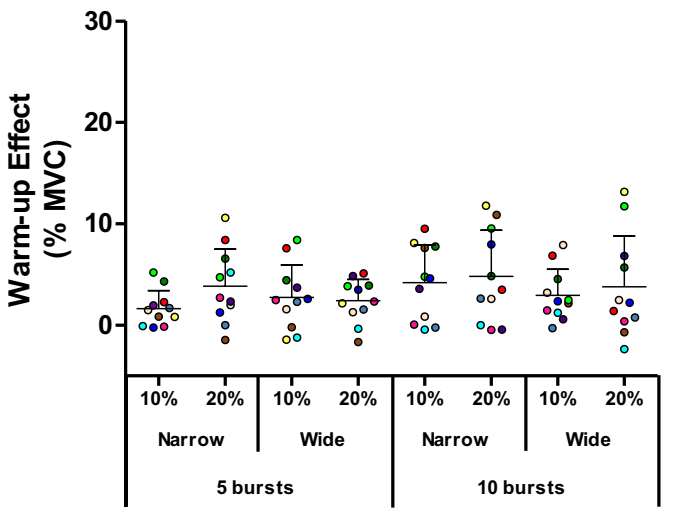

\section{Block 2}

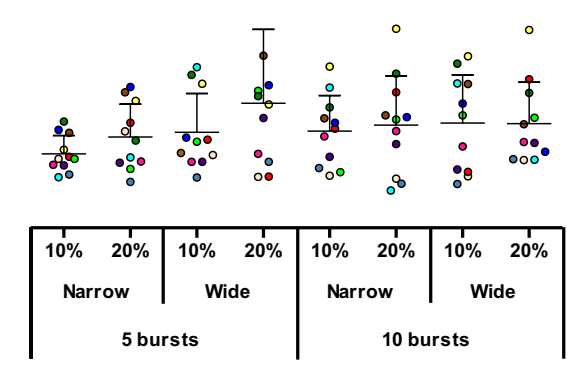

Fig. 3 Magnitude of warm-up in the different conditions. Trains of neuromuscular electrical stimulation (NMES) were applied percutaneously to the plantar flexors with a current intensity to evoke 10 or $20 \%$ of maximal voluntary contraction (MVC) torque, with narrow $(0.2 \mathrm{~ms})$ or wide $(1 \mathrm{~ms})$ pulse widths, in $5 \times 2$-s bursts at 4 -s intervals, or $10 \times 1$-s bursts at 2 -s intervals. Symbols represent data from individuals $(n=11)$, normalized to MVC. Means are shown in horizontal lines with standard deviations indicated. A significant main effect of "intensity" in the ANOVA revealed that a stimulation intensity that evoked $20 \%$ of MVC torque produced a larger warm-up effect than an intensity that evoked 10\% MVC. A significant interaction effect of NMES and pulse width revealed a lower warm-up effect with 5 bursts of narrow pulse-width compared to 10 bursts of narrow pulse-width stimulation
Block 1

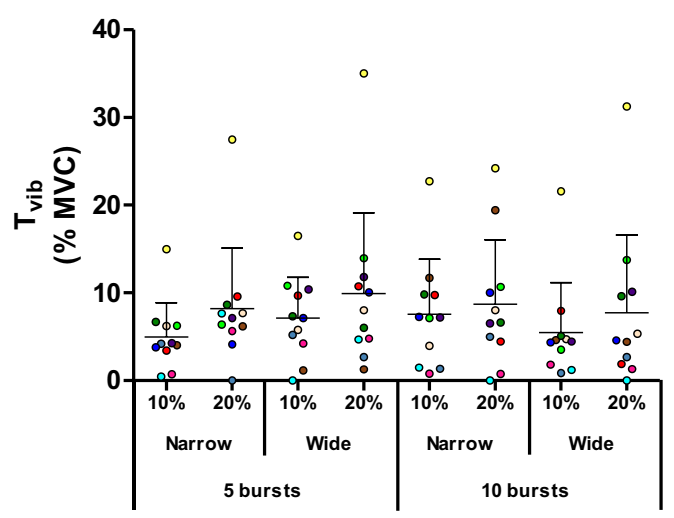

Block 2



Fig. 4 Magnitude of the reflexive torque during vibration $\left(T_{\mathrm{vib}}\right)$ in the different conditions. Trains of NMES were applied percutaneously to the plantar flexors with a current intensity to evoke 10 or $20 \%$ of maximal voluntary contraction (MVC) torque, with narrow $(0.2 \mathrm{~ms})$ or wide $(1 \mathrm{~ms})$ pulse widths, in $5 \times 2$-s bursts at 4 -s intervals, or $10 \times 1$-s bursts at 2 -s intervals. Symbols represent data from individuals $(n=11)$, normalized to MVC. Means are shown in horizontal lines with standard deviations indicated. A significant main effect of "intensity" in the ANOVA revealed that a stimulation intensity that evoked $20 \%$ of MVC torque produced a larger $T_{\text {vib }}$ than an intensity that evoked 10\% MVC. A significant interaction effect of NMES and pulse width revealed a higher $T_{\text {vib }}$ being evoked by 5 bursts of wide pulse-width stimulation than by 5 bursts of narrow pulse-width or by 10 bursts of wide pulse-width stimulation 
Block 1



Block 2

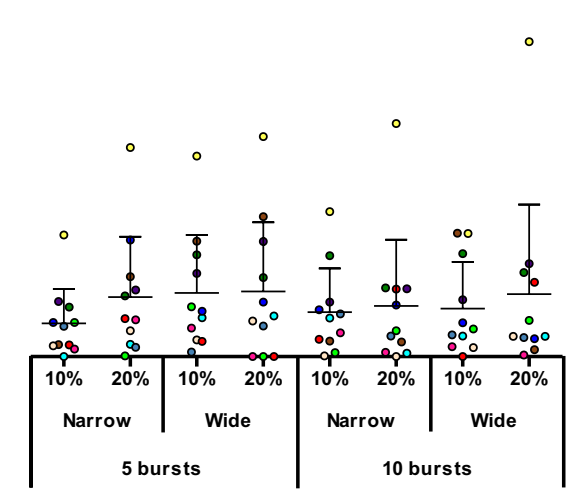

Fig. 5 Magnitude of the sustained torque $\left(T_{\text {sust }}\right)$ in the different conditions. Trains of NMES were applied percutaneously to the plantar flexors with a current intensity to evoke 10 or $20 \%$ of maximal voluntary contraction $(\mathrm{MVC})$ torque, with narrow $(0.2 \mathrm{~ms})$ or wide $(1 \mathrm{~ms})$ pulse widths, in $5 \times 2$-s bursts at 4 -s intervals, or $10 \times 1$-s bursts at

of 20 ( 3 out of 11 responders) perceived this intensity as painful. Hence, analyses were initially conducted without the $30 \%$ MVC condition $(n=11)$, with the results being presented below. Individual data under different experimental conditions can be seen in Fig. 3 (warm-up effect), Fig. $4\left(T_{\text {vib }}\right)$ and Fig. $5\left(T_{\text {sust }}\right)$.

Significant effects of intensity on both warmup $\left[\mathrm{F}(1,10)=8.283, p=0.016, \eta_{\mathrm{p}}{ }^{2}=0.453\right]$ and $T_{\text {vib }}\left[\mathrm{F}(1,10)=9.006, p=0.013, \eta_{\mathrm{p}}{ }^{2}=0.474\right]$ were observed, with both variables being greater in the $20 \%$ MVC than $10 \%$ MVC condition $\left(T_{\mathrm{vib}}: 9.7 \pm 9.0\right.$ vs $7.1 \pm 6.1 \% \mathrm{MVC}$, $p=0.015$; warm-up effect: $4.3 \pm 4.5$ vs $3.7 \pm 3.5 \% \mathrm{MVC}$, $p=0.016)$. No significant effect of intensity was observed for $T_{\text {sust }}(5.3 \pm 6.2$ vs $4.4 \pm 3.9 \% \mathrm{MVC}, p=0.364)$.

Pulse width significantly affected $T_{\text {sust }}[\mathrm{F}(1,10)=6.093$, $\left.p=0.033, \eta_{\mathrm{p}}{ }^{2}=0.379\right]$ but not warm-up (1-ms vs $0.2-\mathrm{ms}$ width: $4.2 \pm 4.5$ vs $3.7 \pm 3.6 \% \mathrm{MVC}, p=0.349)$ or $T_{\text {vib }}$ $(9.2 \pm 8.6$ vs $7.8 \pm 7.0 \% \mathrm{MVC}, p=0.107) . T_{\text {sust }}$ was higher in the 1 -ms than $0.2-\mathrm{ms}$ pulse-width condition $(5.3 \pm 5.9$ vs $4.1 \pm 4.3 \%$ MVC, $p=0.033$ ).

No effect of stimulation pattern $(5 \times 2$-s vs $10 \times 1$-s bursts $)$ was observed on any of the involuntary torque variables 2-s intervals. Symbols represent data from individuals $(n=11)$, normalized to MVC. Means are shown in horizontal lines with standard deviations indicated. A significant main effect of "pulse width" in the ANOVA revealed that wide-pulse NMES evoked a greater $T_{\text {sust }}$ than narrow-pulse NMES

( $T_{\text {vib }}: p=0.267, T_{\text {sust }}: \mathrm{p}=0.925$, warm-up effect: $p=0.239$ ). However, a significant interaction between pattern of stimulation and pulse width was observed for the warmup effect $\left[\mathrm{F}(1,10)=5.143, p=0.047, \eta_{\mathrm{p}}{ }^{2}=0.340\right]$ and $T_{\mathrm{vib}}$ $\left[\mathrm{F}(1,10)=11.799, p=0.006, \eta_{\mathrm{p}}{ }^{2}=0.541\right]$. A significantly higher $T_{\text {vib }}$ was evoked by 5 bursts of wide pulse-width stimulation $(10.6 \pm 9.1 \% \mathrm{MVC})$ than by 5 bursts of narrow pulse-width $(7.4 \pm 7.0 \% \mathrm{MVC}, p=0.003)$ or by 10 bursts of wide pulse-width $(8.0 \pm 7.8 \% \mathrm{MVC}, p=0.012)$. Moreover, a significantly smaller warm-up effect was observed with 5 bursts of narrow pulse-width compared to 10 bursts of narrow pulse-width stimulation $(2.9 \pm 2.8 \%$ vs $4.7 \pm 4.1 \% \mathrm{MVC}$, $p=0.007)$. No other significant interactions were observed.

Overall, the greatest responses were observed in the condition of $5 \times 2$-s bursts at 4 -s intervals with a wide $(1 \mathrm{~ms})$ pulse-width and at an intensity that evoked a torque of $20 \%$ MVC. However, in spite of an absence of a significant difference between sets for any variable $\left(T_{\mathrm{vib}}: p=0.094, T_{\text {sust }}\right.$ : $p=0.234$, warm-up effect: $p=0.109$ ), the level of reliability for this combination of electrical parameters between Set 1 and Set 2 was low (Table 1), with a high degree of variability being observed in some of the participants (Fig. 6).

Table 1 Reliability statistics for differences between Set 1 and Set 2 in the condition of $5 \times 2$-s bursts at 4 -s intervals, with a wide ( 1 ms) pulsewidth, and at an intensity that evoked a torque of $20 \% \mathrm{MVC}$

\begin{tabular}{llllll}
\hline & TE $(95 \%$ CI $)(\%$ MVC $)$ & CV\% & MDC (\%MVC) & MDC (\%) & ICC (95\%) \\
\hline Warm-up & $6(4-11)$ & 78.37 & 27 & 553.61 & $-0.2(-0.569-0.365)$ \\
$T_{\text {vib }}$ & $4(3-7)$ & 58.16 & 15 & 273.36 & $0.178(-0.432-0.682)$ \\
$T_{\text {sust }}$ & $4(3-7)$ & 53.39 & 17 & 115.73 & $0.716(0.232-0.915)$ \\
\hline
\end{tabular}

$T E$ typical error, $C V$ coefficient of variation, $M D C$ minimum detectable change, $I C C$ intraclass correlation coefficient, $T_{v i b}$ and torque after the first burst of NMES, $T_{\text {sust }}$ torque measured $3 \mathrm{~s}$ after vibration cessation (self-sustained torque) 

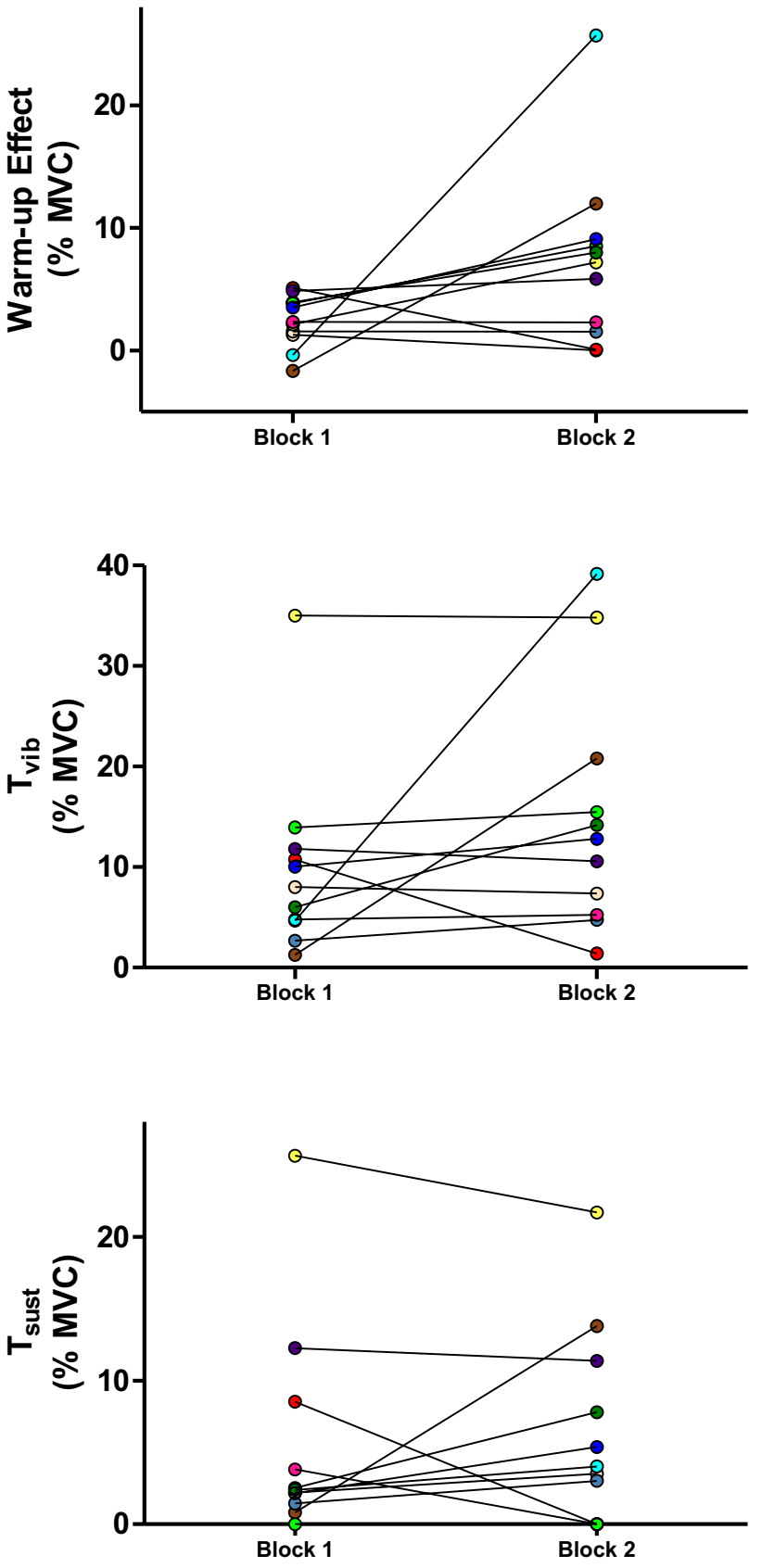

Fig. 6 Individual data of warm-up effect, reflexive torque during vibration $\left(T_{\text {vib }}\right)$ and sustained torque ( $\left.T_{\text {sust }}\right)$ for the two blocks. Symbols represent data from individuals $(n=11)$, normalized to the maximal voluntary contraction torque (MVC), and are shown for the condition which evoked greatest responses. In this condition, trains of neuromuscular electrical stimulation were applied percutaneously to the plantar flexors with a current intensity to elicit $20 \%$ of MVC torque, with wide $(1 \mathrm{~ms})$ pulse-width, in $5 \times 2$-s bursts at 4 -s intervals. Analyses of variance did not reveal a significant difference between blocks for any of the variables, when all the independent factors were taken into consideration. Nevertheless, a high degree of variability was observed in some of the participants
Analyses of variance with the inclusion of $30 \% \mathrm{MVC}$ trials $(n=8)$ only reached significance for $T_{\text {vib }}$, with a significant main effect of intensity $[\mathrm{F}(1.167,8.171)=6.881$, $\left.p=0.027, \eta_{\mathrm{p}}^{2}=0.496\right] . T_{\text {vib }}$ was significantly lower in the $10 \%$ trials $(6.3 \pm 4.4 \%)$ than $20 \%(7.9 \pm 6.2 \%, p=0.011)$ and $30 \%$ MVC trials $(9.7 \pm 7.3 \%, p=0.038)$, with no significant difference between the 20 and $30 \%$ MVC trials $(p=0.507)$.

\section{Discussion}

The aim of the present study was to identify the optimal NMES parameters for use in a method that combines NMES and tendon vibration to evoke involuntary torque. The NMES parameters of intensity, pulse width and pattern of stimulation were systematically tested to determine their effect on the magnitude of increased torque in response to repeated bursts of NMES, the involuntary torque after NMES while the tendon was vibrated, and the sustained torque produced after cessation of tendon vibration. The greatest responses were found with the use of 2-s bursts of NMES every 4 s (i.e. 2 -s rest between bursts) with a wide (1-ms) pulse-width and at an intensity that evoked a torque of $20 \%$ MVC.

In the present study, wide-pulse (1 ms) NMES evoked a greater $T_{\text {sust }}$ than narrow-pulse $(0.2 \mathrm{~ms})$ NMES. These results are consistent with previous findings of greater levels of "extra torque" (Collins et al. 2002; Lagerquist and Collins 2010) and EMG activity (Arpin et al. 2019) during wide- than narrow-pulse NMES. As the membranes of sensory axons have a longer strength-duration time constant and lower rheobase than motor axons (Veale et al. 1973), a higher Ia afferent bombardment of the $\mathrm{MN}$ is likely in the wide-pulse conditions, which probably led to a greater $\mathrm{MN}$ activity.

Stimulation that evoked $20 \%$ of MVC torque produced a larger warm-up effect and $T_{\text {vib }}$ than stimulation that evoked $10 \%$ MVC. Increasing the current intensity applied over the muscle belly produces a stronger depolarising stimulus, which should activate more sensory axons, enhancing the reflex synaptic input to the MN (Zehr 2002). However, increasing current intensity to evoke $30 \%$ MVC did not further augment the observed responses. Speculatively, this might have resulted from greater antidromic collision (Gottlieb and Agarwal 1976; Gregory and Bickel 2005), or from a pain-induced inhibition of MN activity and/or PIC strength (Lund et al. 1991; Farina et al. 2004; Sandrini et al. 2005; Revill and Fuglevand 2017). Taking into consideration the low number of responders who tolerated the $30 \%$ MVC trial in the current study $(n=8)$, further research should confirm that there is no further advantage in increasing intensity to a target level of $30 \% \mathrm{MVC}$ in those who tolerate it well. 
Interrogation of the interaction effect between pattern of stimulation and pulse width revealed that $5 \times 2$-s bursts were superior to $10 \times 1$-s bursts. This is consistent with the findings of Dean et al. (2007), who reported a greater "extra torque" as burst duration increased from 0.25 to $2 \mathrm{~s}$. One possibility is that longer bursts may have allowed a cumulative excitatory effect of Ia afferent bombardment onto the MN, which then recruited more MNs and allowed PICs to grow within each burst. An enhancement of PIC strength within each burst could reflect the slow activation kinetics (Lee and Heckman 1998) and warm-up (Svirskis and Hounsgaard 1997) exhibited by PICs carried by calcium ions. Moreover, given that a torque plateau was not reached in the 2-s condition (Fig. 2), future studies could potentially investigate whether a longer duration of electrical stimulation with fewer bursts would yield similar, or higher, levels of involuntary torque.

It is important to note that the combination of electrical parameters that we recommend evoked responses with greater magnitude but had low levels of reliability between blocks (i.e. between two non-consecutive trials), with the warm-up effect showing the lowest reliability. By contrast, Trajano et al. (2014) showed high levels of reliability of the involuntary torque variables when two trials were performed in succession. The discrepancy between the current results and the findings by Trajano et al. (2014) may be attributed to the performance of 8 or 12 trials, each with different stimulation parameters, between the trials of interest. Numerous factors could contribute to a change in the involuntary torque variables throughout the protocol (as observed in some of the participants, Fig. 6), such as warm-up of PIC amplitude (Svirskis and Hounsgaard 1997), muscle peripheral fatigue (Mettler et al. 2018), decreased efficacy of the Ia afferent$\alpha$-motor neurone pathway (Pope and DeFreitas 2015) and a change in the mental state of the individual throughout the protocol [i.e. more stressed or more relaxed, (Collins et al. 2002)]. While some of these confounding factors were potentially minimised by the 90 -s inter-trial and 10-min inter-block passive rests, their influence cannot be ruled out.

A sustained involuntary torque after the cessation of vibration was not observed in all participants, and some responders showed low levels of involuntary torque. The observation of responders and non-responders has been previously reported in other human studies in which a combination of tendon vibration and NMES (Kirk et al. 2019; Magalhães and Kohn 2010) or NMES only (e.g. Wegrzyk et al. 2015) was imposed. The physiological explanation for this high inter-individual variability is unknown. However several possible mechanisms can be hypothesized, including differences in vibration propagation (Guang et al. 2018), through differences in excitability of the Ia reflex circuitry (Crone and Nielsen 1989; Maffiuletti et al. 2008; Macefield and Knellwolf 2018), to differences in spinal levels of monoamines (Collins 2007; Dean et al. 2007), and the level of relaxation or stress of the participants. However, it should be noted that more non-responders were found in the female cohort, which contrasts the similar proportions of male and female responders observed by Wegrzyk et al. (2015). The potentially higher percentage of female nonresponders should be verified in future studies and the possible causes investigated.

Mechanisms apart from activation of PICs in the MNs may have contributed to the observed involuntary torque enhancement during the test, including pre-synaptic posttetanic potentiation of the Ia input to the MNs (Hirst et al. 1981 ) and modulation of intrinsic muscle properties (Frigon et al. 2011; Blazevich and Babault 2019). However, these mechanisms are unlikely to explain the self-sustained torque in the current study and ongoing soleus EMG activity observed in previous studies (Trajano et al. 2014; Kirk et al. 2019), after the cessation of tendon vibration. One could argue that sustained MN firing could have been caused by the activity in primary muscle spindle afferents as a consequence of thixotropic properties of intrafusal muscle fibres (Kuffler et al. 1951). However, in this technique, a spindle post-contraction sensory discharge is unlikely given that there is no significant background fusimotor drive to spindle endings in the absence of voluntary drive (Gandevia et al. 1986). By contrast, activation of PIC channels to cause bistable behavior in some spinal MNs (Lee and Heckman 1998 ) is a plausible mechanism to explain the observation of sustained involuntary muscle contractions in 11 out of 20 participants after the cessation of tendon vibration. Similarly, the observation of a warm-up effect caused by repetitive activation (i.e. progressive increase in torque within the trial) is also consistent with the activation of PICs in the present protocol (Svirskis and Hounsgaard 1997). Nevertheless, the contribution of PICs is currently unproven. With the advent of new high-density surface EMG techniques, future research could examine the firing of individual MUs during ongoing production of involuntary torque with this technique and potentially examine whether self-sustained firing of MNs, denoting bistable behavior after the cessation of vibration, can be observed (Lee and Heckman, 1998). Moreover, it would be interesting to systematically compare potential changes in the involuntary torque production using this technique with potential changes in $\Delta \mathrm{F}$ values of the paired motor unit technique (Gorassini et al. 2002) during voluntary contractions, as the latter technique is presently the standard method to indirectly estimate PIC strength in humans. To date, acute static stretching is one intervention that has been observed to reduce both involuntary plantar flexion torque production (Trajano et al. 2014) and soleus $\Delta \mathrm{F}$ values (Trajano et al. 2020), indicating that both techniques may provide similar information. If tendon vibration and NMES can be established as a measure of PICs, it 
should be noted that not everyone responds to the stimulus and some participants have a low magnitude of involuntary torque, which might impact the widespread use of this methodological approach.

A limitation of the current study is that the Achilles tendon was mechanically vibrated at $115 \mathrm{~Hz}$ with $1-\mathrm{mm}$ deflections by an experienced researcher, but the pressure on the skin was not quantified. It is possible that small pressure variations might have partly contributed to the observed variability between blocks. Finally, future research could quantify whether higher-frequency trains of NMES would have resulted in involuntary torque with higher amplitude (Dean et al. 2007), given that it might further enhance the effective current delivered to MNs along the sensory axons. However, higher stimulation frequencies may both increase the risk of peripheral fatigue during a long experimental protocol (Grosprêtre et al. 2017) and variably influence neurotransmitter release from Ia afferent terminals (Hirst et al. 1981; Crone and Nielsen 1989).

\section{Conclusion}

A combination of Achilles tendon vibration with superimposed NMES on triceps surae induces involuntary sustained torque in some individuals. Tailoring NMES parameters modulates the magnitude of the involuntary torque, and we observed that a current intensity to evoke $20 \%$ of MVC torque with wide $(1 \mathrm{~ms})$ pulse-width in $5 \times 2$-s bursts and at 4-s intervals produces the greatest responses and therefore is recommended for use with this technique. These findings may also inform the parameters of percutaneous electrical stimulation used in other clinical and experimental settings.

Author contributions All authors contributed to the study conception and design. RNOM completed data collection and analysis. The first draft of the manuscript was written by RNOM and all authors commented on previous versions of the manuscript. All authors read and approved the final manuscript.

Availability of data and materials Non-identifiable datasets generated and/or analyzed during the current study are available from the corresponding author on reasonable request.

Code availability Macros for semi-automated analysis of torque data in LabChart utilized in this study are available from the corresponding author on reasonable request.

\section{Compliance with ethical standards}

Conflicts of interest There are no conflicts of interest, financial or otherwise to report regarding this manuscript.

Ethics approval Participants were fully informed of any risks or discomforts associated with the procedures before giving their written informed consent to participate. The procedures were approved by the Human Research Ethics Committee of Edith Cowan University, and performed according to the Declaration of Helsinki, except for registration in a database.

Open Access This article is licensed under a Creative Commons Attribution 4.0 International License, which permits use, sharing, adaptation, distribution and reproduction in any medium or format, as long as you give appropriate credit to the original author(s) and the source, provide a link to the Creative Commons licence, and indicate if changes were made. The images or other third party material in this article are included in the article's Creative Commons licence, unless indicated otherwise in a credit line to the material. If material is not included in the article's Creative Commons licence and your intended use is not permitted by statutory regulation or exceeds the permitted use, you will need to obtain permission directly from the copyright holder. To view a copy of this licence, visit http://creativecommons.org/licenses/by/4.0/.

\section{References}

Arpin DJ, Ugiliweneza B, Forrest G, Harkema SJ, Rejc E (2019) Optimizing neuromuscular electrical stimulation pulse width and amplitude to promote central activation in individuals with severe spinal cord injury. Front Physiol 10:1310. https://doi.org/10.3389/ fphys.2019.01310

Bergquist AJ, Clair JM, Collins DF (2011a) Motor unit recruitment when neuromuscular electrical stimulation is applied over a nerve trunk compared with a muscle belly: triceps surae. J Appl Physiol 110:627-637. https://doi.org/10.1152/japplphysiol.01103.2010

Bergquist AJ, Clair JM, Lagerquist O, Mang CS, Okuma Y, Collins DF (2011b) Neuromuscular electrical stimulation: implications of the electrically evoked sensory volley. Eur J Appl Physiol 111:2409_ 2426. https://doi.org/10.1007/s00421-011-2087-9

Blazevich AJ, Babault N (2019) Post-activation potentiation (PAP) versus post-activation performance enhancement (PAPE) in humans: historical perspective, underlying mechanisms, and current issues. Front Physiol 10:1359. https://doi.org/10.3389/FPHYS .2019 .01359

Blouin JS, Walsh LD, Nickolls P, Gandevia SC (2009) High-frequency submaximal stimulation over muscle evokes centrally generated forces in human upper limb skeletal muscles. J Appl Physiol 106:370-377. https://doi.org/10.1152/japplphysiol.90939.2008

Bochkezanian V, Newton RU, Trajano GS, Vieira A, Pulverenti TS, Blazevich AJ (2018a) Effect of tendon vibration during widepulse neuromuscular electrical stimulation (NMES) on muscle force production in people with spinal cord injury (SCI). BMC Neurol 18(1):17. https://doi.org/10.1186/s12883-018-1020-9

Bochkezanian V, Newton RU, Trajano GS, Blazevich AJ (2018b) Effects of neuromuscular electrical stimulation in people with spinal cord injury. Med Sci Sports Exerc 50(9):1733-1739. https ://doi.org/10.1249/MSS.0000000000001637 
Collins DF (2007) Central contributions to contractions evoked by tetanic neuromuscular electrical stimulation. Exerc Sport Sci Rev 35(3):102-109. https://doi.org/10.1097/jes.0b013e3180a0321b

Collins DF, Burke D, Gandevia SC (2001) Large involuntary forces consistent with plateau-like behavior of human motoneurons. J Neurosci 21:4059-4065. https://doi.org/10.1523/JNEUROSCI.2111-04059.2001

Collins DF, Burke D, Gandevia SC (2002) Sustained contractions produced by plateau-like behaviour in human motoneurones. J Physiol 538:289-301. https://doi.org/10.1113/jphysiol.2001.012825

Crone C, Nielsen J (1989) Methodological implications of the post activation depression of the soleus H-reflex in man. Exp Brain Res 78:28-32. https://doi.org/10.1007/BF00230683

Dean JC, Yates LM, Collins DF (2007) Turning on the central contribution to contractions evoked by neuromuscular electrical stimulation. J Appl Physiol 103:170-176. https://doi.org/10.1152/jappl physiol.01361.2006

Farina D, Arendt-Nielsen L, Merletti R, Graven-Nielsen T (2004) Effect of experimental muscle pain on motor unit firing rate and conduction velocity. J Neurophysiol 91:1250-1259. https://doi. org/10.1152/jn.00620.2003

Frigon A, Thompson CK, Johnson MD, Manuel M, Hornby TG, Heckman CJ (2011) Extra forces evoked during electrical stimulation of the muscle or its nerve are generated and modulated by a length-dependent intrinsic property of muscle in humans and cats. J Neurosci 31:5579-5588. https://doi.org/10.1523/JNEUR OSCI.6641-10.2011

Gandevia SC, Miller S, Aniss AM, Burke D (1986) Reflex influences on muscle spindle activity in relaxed human leg muscles. J Neurophysiol 56:159-170. https://doi.org/10.1152/jn.1986.56.1.159

Gorassini MA, Yang JF, Siu M, Bennett DJ (2002) Intrinsic activation of human motoneurons: Possible contribution to motor unit excitation. J Neurophysiol 87:1850-1858. https://doi.org/10.1152/jn.00024 .2001

Gottlieb GL, Agarwal GC (1976) Extinction of the Hoffman reflex by antidromic conduction. Electroencephalogr Clin Neurophysiol 41:19-24. https://doi.org/10.1016/0013-4694(76)90211-X

Grande G, Cafarelli E (2003) Ia afferent input alters the recruitment thresholds and firing rates of single human motor units. Exp Brain Res 150:449-457. https://doi.org/10.1007/s00221-003-1463-Z

Gregory CM, Bickel CS (2005) Recruitment patterns in human skeletal muscle during electrical stimulation. Phys Ther 85:358-364. https ://doi.org/10.1093/ptj/85.4.358

Grosprêtre S, Gueugneau N, Martin A, Lepers R (2017) Central contribution to electrically induced fatigue depends on stimulation frequency. Med Sci Sports Exerc 49:1530-1540. https://doi.org/10.1249/ MSS.0000000000001270

Guang H, Ji L, Shi Y (2018) Focal vibration stretches muscle fibers by producing muscle waves. IEEE Trans Neural Syst Rehabil Eng 26:839-846. https://doi.org/10.1109/TNSRE.2018.2816953

Heckman CJ, Gorassini MA, Bennett DJ (2005) Persistent inward currents in motoneuron dendrites: Implications for motor output. Muscle Nerve 31:135-156. https://doi.org/10.1002/mus.20261

Heckman CJ, Johnson M, Mottram C, Schuster J (2008) Persistent inward currents in spinal motoneurons and their influence on human motoneuron firing patterns. Neuroscientist 14:264-275. https://doi. org/10.1177/1073858408314986

Hirst GD, Redman SJ, Wong K (1981) Post-tetanic potentiation and facilitation of synaptic potentials evoked in cat spinal motoneurones. J Physiol 321:97-109. https://doi.org/10.1113/jphysiol.1981.sp013 973

Hopkins W (2017) Spreadsheets for analysis of validity and reliability. Sportscience 21:36-44

Hounsgaard J, Kiehn O (1993) Calcium spikes and calcium plateaux evoked by differential polarization in dendrites of turtle motoneurones in vitro. J Physiol 468:245-259. https://doi. org/10.1113/jphysiol.1993.sp019769

Hyngstrom AS, Johnson MD, Miller JF, Heckman CJ (2007) Intrinsic electrical properties of spinal motoneurons vary with joint angle. Nat Neurosci 10:363-369. https://doi.org/10.1038/nn1852

Kirk BJC, Trajano GS, Pulverenti TS, Rowe G, Blazevich AJ (2019) Neuromuscular factors contributing to reductions in muscle force after repeated, high-intensity muscular efforts. Front Physiol 10:1-17. https://doi.org/10.3389/fphys.2019.00783

Klakowicz PM, Baldwin ERL, Collins DF (2006) Contribution of M-waves and H-reflexes to contractions evoked by tetanic nerve stimulation in humans. J Neurophysiol 96:1293-1302. https://doi. org/10.1152/jn.00765.2005

Kuffler SW, Hunt CC, Quilliam JP (1951) Function of medullated smallnerve fibers in mammalian ventral roots; efferent muscle spindle innervation. J Neurophysiol 14:29-54. https://doi.org/10.1152/ jn.1951.14.1.29

Lagerquist O, Collins DF (2010) Influence of stimulus pulse width on $\mathrm{M}$-waves, H-reflexes, and torque during tetanic low-intensity neuromuscular stimulation. Muscle Nerve 42:886-893. https://doi. org/10.1002/mus.21762

Lagerquist O, Walsh LD, Blouin JS, Collins DF, Gandevia SC (2009) Effect of a peripheral nerve block on torque produced by repetitive electrical stimulation. J Appl Physiol 107:161-167. https://doi. org/10.1152/japplphysiol.91635.2008

Lee RH, Heckman CJ (1996) Influence of voltage-sensitive dendritic conductances on bistable firing and effective synaptic current in cat spinal motoneurons in vivo. J Neurophysiol 76:2107-2110. https:// doi.org/10.1152/jn.1996.76.3.2107

Lee RH, Heckman CJ (1998) Bistability in spinal motoneurons in vivo: systematic variations in persistent inward currents. J Neurophysiol 80:583-593. https://doi.org/10.1152/jn.1998.80.2.583

Lee RH, Heckman CJ (2000) Adjustable amplification of synaptic input in the dendrites of spinal motoneurons in vivo. J Neurosci 20:6734 6740. https://doi.org/10.1523/JNeurosci.20-17-06734.2000

Lund JP, Donga R, Widmer CG, Stohler CS (1991) The pain-adaptation model: a discussion of the relationship between chronic musculoskeletal pain and motor activity. Can J Physiol Pharmacol 69(5):683-694

Macefield VG, Knellwolf TP (2018) Functional properties of human muscle spindles. J Neurophysiol 120:452-467. https://doi.org/10.1152/ jn.00071.2018

Maffiuletti NA, Herrero AJ, Jubeau M, Impellizzeri FM, Bizzini M (2008) Differences in electrical stimulation thresholds between men and women. Ann Neurol 63:507-512. https://doi.org/10.1002/ ana. 21346

Magalhães FH, Kohn AF (2010) Vibration-induced extra torque during electrically-evoked contractions of the human calf muscles. $\mathrm{J}$ Neuroeng Rehabil 7(1):26. https://doi.org/10.1186/1743-0003-7-26

Magalhães FH, De Toledo DR, Kohn AF (2013) Plantar flexion force induced by amplitude-modulated tendon vibration and associated soleus V/F-waves as an evidence of a centrally-mediated mechanism contributing to extra torque generation in humans. J Neuroeng Rehabil 10(1):32. https://doi.org/10.1186/1743-0003-10-32

Mettler JA, Magee DM, Doucet BM (2018) Low-frequency electrical stimulation with variable intensity preserves torque. J Electromyogr Kinesiol 42:49-56. https://doi.org/10.1016/j.jelekin.2018.06.007

Pope ZK, DeFreitas JM (2015) The effects of acute and prolonged muscle vibration on the function of the muscle spindle's reflex arc. Somatosens Mot Res 32:254-261. https://doi.org/10.3109/08990 220.2015.1091770

Revill AL, Fuglevand AJ (2017) Inhibition linearizes firing rate responses in human motor units: implications for the role of persistent inward currents. J Physiol 595:179-191. https://doi.org/10.1113/JP272823 
Sandrini G, Serrao M, Rossi P, Romaniello A, Cruccu G, Willer JC (2005) The lower limb flexion reflex in humans. Prog Neurobiol 77:353-395. https://doi.org/10.1016/j.pneurobio.2005.11.003

Svirskis G, Hounsgaard J (1997) Depolarization-induced facilitation of a plateau-generating current in ventral horn neurons in the turtle spinal cord. J Neurophysiol 78:1740-1742. https://doi.org/10.1152/ jn.1997.78.3.1740

Trajano GS, Seitz LB, Nosaka K, Blazevich AJ (2014) Can passive stretch inhibit motoneuron facilitation in the human plantar flexors? J Appl Physiol 117:1486-1492. https://doi.org/10.1152/japplphysiol.00809 .2014

Trajano GS, Taylor JL, Orssatto LB, McNulty CR, Blazevich AJ (2020) Passive muscle stretching reduces estimates of persistent inward current strength in soleus motor units. J Exp Biol. https://doi. org/10.1242/jeb.229922

Veale JL, Mark RF, Rees S (1973) Differential sensitivity of motor and sensory fibres in human ulnar nerve. J Neurol Neurosurg Psychiatry 36:75-86. https://doi.org/10.1136/jnnp.36.1.75
Vitry F, Martin A, Deley G, Papaiordanidou M (2019) Effect of reflexive activation of motor units on torque development during electricallyevoked contractions of the triceps surae muscle. J Appl Physiol 126:386-392. https://doi.org/10.1152/japplphysiol.00463.2018

Wegrzyk J, Fouré A, Vilmen C, Ghattas B, Maffiuletti NA, Mattei JP, Place N, Bendahan D, Gondin J (2015) Extra Forces induced by wide-pulse, high-frequency electrical stimulation: Occurrence, magnitude, variability and underlying mechanisms. Clin Neurophysiol 126:1400-1412. https://doi.org/10.1016/j.clinph.2014.10.001

Zehr EP (2002) Considerations for use of the Hoffmann reflex in exercise studies. Eur J Appl Physiol 86:455-468. https://doi.org/10.1007/ s00421-002-0577-5

Publisher's Note Springer Nature remains neutral with regard to jurisdictional claims in published maps and institutional affiliations. 